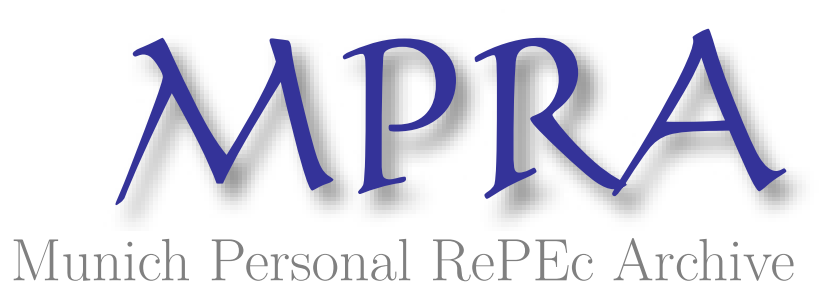

\title{
Economic growth and debt: a simplified model
}

Krouglov, Alexei

21 June 2015

Online at https://mpra.ub.uni-muenchen.de/65178/

MPRA Paper No. 65178, posted 22 Jun 2015 06:14 UTC 


\title{
Economic Growth and Debt: A Simplified Model
}

\author{
Alexei Krouglov
}

alexkrouglov@gmail.com 


\title{
Economic Growth and Debt: A Simplified Model
}

\begin{abstract}
Presented is a mathematical model of single-product economy where an investment and debt are used to alter the demand for and supply of product. Explored is the dynamics of a nominal economic growth and decline. Examined are cases of a constant-rate growing debt and a constant-rate and constant-acceleration growing investment.
\end{abstract}

JEL Classification Numbers: E32, E51, E22

Keywords: debt, investment, modeling 


\section{Introduction}

There are four enormously important concepts in the economics: demand, supply, investment, and debt. Here I present a mathematical model, where I examine their interconnections and explore their impacts on the nominal economic growth and decline.

Below I describe a mathematical model of the market of single-product economy. Economic forces acting on the market represent inherent market forces of demand and supply complemented with the forces caused by an investment, debt and corresponding debt servicing. The market actions are expressed through the system of ordinary differential equations.

The model continues prior research for nominal economic growth and decline performed in Krouglov, 2014 and previous research for credit expansion and contraction carried out in Krouglov, 2015.

The presented model has mostly a theoretical value.

\section{Single-Product Economy at the Undisturbed State}

Concepts and methodology presented in this section are based on the framework of mathematical dynamics of economic systems developed in Krouglov, 2006; 2009.

When there are no disturbing economic forces, the market is in equilibrium position, i.e., the supply of and demand for product are equal, they are developing with a constant rate and a price of the product is fixed.

I assume the market had been in an equilibrium until time $t=t_{0}$, volumes of the product supply $V_{S}(t)$ and demand $V_{D}(t)$ on market were equal, and they both were developing with a constant rate $r_{D}^{0}$. The product price $P(t)$ at that time was fixed, 
$V_{D}(t)=r_{D}^{0}\left(t-t_{0}\right)+V_{D}^{0}$

$V_{S}(t)=V_{D}(t)$

$P(t)=P^{0}$

where $V_{D}\left(t_{0}\right)=V_{D}^{0}$.

When the balance between the volumes of product supply and demand is broken, a market is experiencing economic forces, which act to bring the market to a new equilibrium position.

\section{Constant-Rate Debt and Constant-Rate Investment}

I present a model of single-product economy where both the credit and investment are increasing with constant rates.

According to this scenario, the credit expansion causes a debt growth where the amount of debt $S_{D}(t)$ on the market rises since time $t=t_{0}$ according to following formula,

$S_{D}(t)=\left\{\begin{array}{cc}0, & t<t_{0} \\ \delta_{D}\left(t-t_{0}\right), & t \geq t_{0}\end{array}\right.$

where $S_{D}(t)=0$ for $t<t_{0}$ and $\delta_{D}>0$.

Correspondingly, the debt growth causes an increase of debt servicing cost $s_{S}(t)=\delta_{S} S_{D}(t), \delta_{S}>0$, where the accumulated amount of debt servicing cost $S_{S}(t)$ on the market rises according to following formula since time $t=t_{0}$,

$S_{S}(t)=\left\{\begin{array}{cc}0, & t<t_{0} \\ \frac{\delta_{S} \delta_{D}}{2}\left(t-t_{0}\right)^{2}, & t \geq t_{0}\end{array}\right.$ 
where $S_{S}(t)=0$ for $t<t_{0}$ and $\delta_{S}>0$.

Likewise, I assume the amount of investment $S_{I}(t)$ on the market increases since time $t=t_{0}$ according to following formula,

$S_{I}(t)=\left\{\begin{array}{cc}0, & t<t_{0} \\ \delta_{I}\left(t-t_{0}\right), & t \geq t_{0}\end{array}\right.$

where $S_{I}(t)=0$ for $t<t_{0}$ and $\delta_{I}>0$.

Economic forces trying to bring the market into a new equilibrium position are described by the following ordinary differential equations with regard to the volumes of product supply $V_{S}(t)$, demand $V_{D}(t)$, and price $P(t)$ given the accumulated amounts of debt $S_{D}(t)$, debt servicing cost $S_{S}(t)$, and investment $S_{I}(t)$ on the market (see Krouglov, 2006; 2009; 2014; 2015),

$\frac{d P(t)}{d t}=-\lambda_{P}\left(V_{S}(t)-V_{D}(t)-S_{D}(t)+S_{S}(t)-S_{I}(t)\right)$

$\frac{d^{2} V_{S}(t)}{d t^{2}}=\lambda_{S} \frac{d P(t)}{d t}$

$\frac{d^{2} V_{D}(t)}{d t^{2}}=-\lambda_{D} \frac{d^{2} P(t)}{d t^{2}}$

In Eqs. (7) - (9) above the values $\lambda_{P}, \lambda_{S}, \lambda_{D} \geq 0$ are constants and they characterize price inertness, supply inducement, and demand amortization correspondingly.

One may observe that a difference between the volumes of product demand and supply on the market is increasing, in the sense:

$$
V_{D}(t)-V_{S}(t)+\left(S_{I}(t)+S_{D}(t)-S_{S}(t)\right) \geq V_{D}(t)-V_{S}(t)
$$

if the sum of accumulated amount of investment $S_{I}(t)$ and accumulated amount of debt $S_{D}(t)$ exceeds the accumulated amount of debt servicing cost $S_{S}(t): S_{I}(t)+S_{D}(t) \geq S_{S}(t)$. 
On the other hand, a difference between the volumes of product demand and supply on the market is decreasing, in the sense:

$$
V_{D}(t)-V_{S}(t)+\left(S_{I}(t)+S_{D}(t)-S_{S}(t)\right)<V_{D}(t)-V_{S}(t)
$$

if the sum of accumulated amount of investment $S_{I}(t)$ and accumulated amount of debt $S_{D}(t)$ goes below the accumulated amount of debt servicing cost $S_{S}(t): S_{I}(t)+S_{D}(t)<S_{S}(t)$.

Thus, in the time interval $t_{0} \leq t \leq t_{0}+\frac{2\left(\delta_{I}+\delta_{D}\right)}{\delta_{S} \delta_{D}}$ an expansion of the product demand over supply takes place, and in the time interval $t_{0}+\frac{2\left(\delta_{I}+\delta_{D}\right)}{\delta_{S} \delta_{D}}<t<+\infty$ a contraction of the product demand over supply happens.

Let me introduce a new variable $D(t) \equiv\left(V_{S}(t)-V_{D}(t)-S_{D}(t)+S_{S}(t)-S_{I}(t)\right)$ representing the volume of product surplus (or shortage) on the market. Therefore, behavior of $D(t)$ is described by the following equation for $t>t_{0}$,

$\frac{d^{2} D(t)}{d t^{2}}+\lambda_{P} \lambda_{D} \frac{d D(t)}{d t}+\lambda_{P} \lambda_{S} D(t)-\delta_{S} \delta_{D}=0$

with the initial conditions, $D\left(t_{0}\right)=0, \frac{d D\left(t_{0}\right)}{d t}=-\delta_{I}-\delta_{D}$.

If one uses another variable $D_{1}(t) \equiv D(t)-\frac{\delta_{S} \delta_{D}}{\lambda_{P} \lambda_{S}}$, then Eq. (10) becomes,

$\frac{d^{2} D_{1}(t)}{d t^{2}}+\lambda_{P} \lambda_{D} \frac{d D_{1}(t)}{d t}+\lambda_{P} \lambda_{S} D_{1}(t)=0$

with the initial conditions, $D_{1}\left(t_{0}\right)=-\frac{\delta_{S} \delta_{D}}{\lambda_{P} \lambda_{S}}, \frac{d D_{1}\left(t_{0}\right)}{d t}=-\delta_{I}-\delta_{D}$. 
Similar to Eq. (10), the product price $P(t)$ is described by the following equation for $t>t_{0}$,

$$
\frac{d^{2} P(t)}{d t^{2}}+\lambda_{P} \lambda_{D} \frac{d P(t)}{d t}+\lambda_{P} \lambda_{S}\left(P(t)-P^{0}-\frac{\delta_{I}+\delta_{D}}{\lambda_{S}}+\frac{\delta_{S} \delta_{D}}{\lambda_{S}}\left(t-t_{0}\right)\right)=0
$$

with the initial conditions, $P\left(t_{0}\right)=P^{0}, \frac{d P\left(t_{0}\right)}{d t}=0$.

Let me introduce variable $P_{1}(t) \equiv P(t)-P^{0}-\frac{\delta_{I}+\delta_{D}}{\lambda_{S}}+\frac{\delta_{S} \delta_{D}}{\lambda_{S}}\left(t-t_{0}\right)-\frac{\lambda_{D}}{\lambda_{S}^{2}} \delta_{S} \delta_{D}$ to simplify an analysis of the product price behavior. The behavior of variable $P_{1}(t)$ is described by the equation for $t>t_{0}$,

$$
\frac{d^{2} P_{1}(t)}{d t^{2}}+\lambda_{P} \lambda_{D} \frac{d P_{1}(t)}{d t}+\lambda_{P} \lambda_{S} P_{1}(t)=0
$$

with the initial conditions, $P_{1}\left(t_{0}\right)=-\frac{\delta_{I}+\delta_{D}}{\lambda_{S}}-\frac{\lambda_{D}}{\lambda_{S}^{2}} \delta_{S} \delta_{D}, \frac{d P_{1}\left(t_{0}\right)}{d t}=\frac{\delta_{S} \delta_{D}}{\lambda_{S}}$

The behavior of solutions for $D_{1}(t)$ and $P_{1}(t)$ described by Eqs. (11) and (13) depends on the roots of the corresponding characteristic equations (Piskunov, 1965; Petrovski, 1966). Note that Eqs. (11) and (13) have the same characteristic equations.

When the roots of characteristic equation are complex-valued (i.e., $\frac{\lambda_{P}^{2} \lambda_{D}^{2}}{4}<\lambda_{P} \lambda_{S}$ ) both the variable $D_{1}(t)$ and variable $P_{1}(t)$ experience damped oscillations for time $t \geq t_{0}$. When the roots of characteristic equation are real and different (i.e., $\left.\frac{\lambda_{P}^{2} \lambda_{D}^{2}}{4}>\lambda_{P} \lambda_{S}\right)$ both the variable $D_{1}(t)$ and variable $P_{1}(t)$ don't 
oscillate for time $t \geq t_{0}$. When the roots of characteristic equation are real and equal (i.e., $\left.\frac{\lambda_{P}^{2} \lambda_{D}^{2}}{4}=\lambda_{P} \lambda_{S}\right)$ both the variable $D_{1}(t)$ and variable $P_{1}(t)$ don't oscillate for time $t \geq t_{0}$ as well.

It takes place $D_{1}(t) \rightarrow 0$ and $P_{1}(t) \rightarrow 0$ for $t \rightarrow+\infty$ if roots of characteristic equations are complexvalued $\left(\frac{\lambda_{P}^{2} \lambda_{D}^{2}}{4}<\lambda_{P} \lambda_{S}\right)$, real and different $\left(\frac{\lambda_{P}^{2} \lambda_{D}^{2}}{4}>\lambda_{P} \lambda_{S}\right)$, or real and equal $\left(\frac{\lambda_{P}^{2} \lambda_{D}^{2}}{4}=\lambda_{P} \lambda_{S}\right)$.

It takes place for the product surplus (shortage) $D(t)$, for the product price $P(t)$, for the product demand $V_{D}(t)$, for the product supply $V_{S}(t)$, for the amount of debt $S_{D}(t)$, for the amount of debt servicing cost $S_{S}(t)$, and for the amount of investment $S_{I}(t)$ if $t \rightarrow+\infty$,

$$
D(t) \rightarrow \frac{\delta_{S} \delta_{D}}{\lambda_{P} \lambda_{S}}
$$

$P(t) \rightarrow-\frac{\delta_{S} \delta_{D}}{\lambda_{S}}\left(t-t_{0}\right)+P^{0}+\frac{\delta_{I}+\delta_{D}}{\lambda_{S}}+\frac{\lambda_{D}}{\lambda_{S}^{2}} \delta_{S} \delta_{D}$

$V_{D}(t) \rightarrow\left(r_{D}^{0}+\frac{\lambda_{D}}{\lambda_{S}} \delta_{S} \delta_{D}\right)\left(t-t_{0}\right)+V_{D}^{0}-\frac{\lambda_{D}}{\lambda_{S}}\left(\delta_{I}+\delta_{D}\right)-\frac{\lambda_{D}^{2}}{\lambda_{S}^{2}} \delta_{S} \delta_{D}$

$V_{S}(t) \rightarrow\left(r_{D}^{0}+\delta_{I}+\delta_{D}+\frac{\lambda_{D}}{\lambda_{S}} \delta_{S} \delta_{D}\right)\left(t-t_{0}\right)-\frac{\delta_{S} \delta_{D}}{2}\left(t-t_{0}\right)^{2}+V_{D}^{0}-\frac{\lambda_{D}}{\lambda_{S}}\left(\delta_{I}+\delta_{D}\right)$

$+\frac{\delta_{S} \delta_{D}}{\lambda_{P} \lambda_{S}}-\frac{\lambda_{D}^{2}}{\lambda_{S}^{2}} \delta_{S} \delta_{D}$

$S_{I}(t)=\delta_{I}\left(t-t_{0}\right)$

$S_{D}(t)=\delta_{D}\left(t-t_{0}\right)$

$S_{S}(t)=\frac{\delta_{S} \delta_{D}}{2}\left(t-t_{0}\right)^{2}$ 
To analyze an economic growth I use the variable $E_{D}(t) \equiv P(t) \times r_{D}(t)$ where $r_{D}(t) \equiv \frac{d V_{D}(t)}{d t}$, i.e., a rate of nominal demand for the product, which roughly represents the product earning on the market.

I compare the variable $E_{D}(t)$, rate of nominal demand changed by the amount of debt $S_{D}(t)$, the amount of debt servicing cost $S_{S}(t)$, and the amount of investment $S_{I}(t)$, with the variable $\widetilde{E}_{D}(t)$, original rate unchanged by the amounts of debt, debt servicing cost and investment, for $t \rightarrow+\infty$, $E_{D}(t) \rightarrow\left(-\frac{\delta_{S} \delta_{D}}{\lambda_{S}}\left(t-t_{0}\right)+P^{0}+\frac{\delta_{I}+\delta_{D}}{\lambda_{S}}+\frac{\lambda_{D}}{\lambda_{S}^{2}} \delta_{S} \delta_{D}\right)\left(r_{D}^{0}+\frac{\lambda_{D}}{\lambda_{S}} \delta_{S} \delta_{D}\right)$ and $\tilde{E}_{D}(t) \rightarrow P^{0} r_{D}^{0}$

Thus, if the amounts of debt $S_{D}(t)$ and of investment $S_{I}(t)$ are correspondingly increasing with a constant-rate $\delta_{D}>0$ and a constant-rate $\delta_{I}>0$ to advance an expansion of the product demand over supply on the market then the amount of debt servicing cost $S_{S}(t)$ is increasing with a constantacceleration $\delta_{S} \delta_{D}>0$ and ultimately causing an unrestricted decrease of the rate of nominal demand $E_{D}(t)$ with the passage of time.

We can estimate a decrease $e_{D}(t)$ of the rate of nominal demand $E_{D}(t)$ where $e_{D}(t) \equiv \frac{d E_{D}(t)}{d t}$, i.e., the decrease of the rate of nominal demand for product, which roughly represents the decrease of the product earning on market.

It takes place, for $t \rightarrow+\infty, e_{D}(t) \rightarrow-\frac{\delta_{S} \delta_{D}}{\lambda_{S}}\left(r_{D}^{0}+\frac{\lambda_{D}}{\lambda_{S}} \delta_{S} \delta_{D}\right)<0$ when $\delta_{S} \delta_{D}>0$. 
The limitary value of variable $e_{D}(t)$ doesn't have extremal points in the region $\delta_{S} \delta_{D}>0$. In fact, the variable $e_{D}(t)$ has maximal limitary value when $\delta_{S} \delta_{D}=-\frac{\lambda_{S}}{2 \lambda_{D}} r_{D}^{0}$. Then, respectively for $t \rightarrow+\infty$, $\max \left\{e_{D}(t)\right\} \rightarrow \frac{1}{4 \lambda_{D}}\left(r_{D}^{0}\right)^{2}>0$

Therefore, the limitary value of variable $e_{D}(t)$ is always negative in the region $\delta_{S} \delta_{D}>0$. Maximum for the change of a rate of nominal demand for product, $\max \left\{e_{D}(t)\right\}$, is achieved outside of the region $\delta_{S} \delta_{D}>0$, and is equal at the limit, for time $t \rightarrow+\infty, \max \left\{e_{D}(t)\right\} \rightarrow \frac{1}{4 \lambda_{D}}\left(r_{D}^{0}\right)^{2}>0$

I will talk about economic implications of some results in a succeeding section.

\section{Constant-Rate Debt and Constant-Acceleration Investment}

I present a model of single-product economy where the credit is increasing with a constant rate and the investment is increasing with a constant acceleration.

According to this scenario, the credit expansion causes a debt growth where the amount of debt $S_{D}(t)$ on the market rises since time $t=t_{0}$ according to following formula,

$S_{D}(t)=\left\{\begin{array}{cc}0, & t<t_{0} \\ \delta_{D}\left(t-t_{0}\right), & t \geq t_{0}\end{array}\right.$

where $S_{D}(t)=0$ for $t<t_{0}$ and $\delta_{D}>0$. 
Correspondingly, the debt growth causes an increase of debt servicing cost $s_{S}(t)=\delta_{S} S_{D}(t), \delta_{S}>0$, where the accumulated amount of debt servicing cost $S_{S}(t)$ on the market rises according to following formula since time $t=t_{0}$,

$S_{S}(t)=\left\{\begin{array}{cc}0, & t<t_{0} \\ \frac{\delta_{S} \delta_{D}}{2}\left(t-t_{0}\right)^{2}, & t \geq t_{0}\end{array}\right.$

where $S_{S}(t)=0$ for $t<t_{0}$ and $\delta_{S}>0$.

Likewise, I assume the amount of investment $S_{I}(t)$ on the market increases since time $t=t_{0}$ according to following formula,

$S_{I}(t)=\left\{\begin{array}{cc}0, & t<t_{0} \\ \delta_{I}\left(t-t_{0}\right)+\frac{\varepsilon_{I}}{2}\left(t-t_{0}\right)^{2}, & t \geq t_{0}\end{array}\right.$

where $S_{I}(t)=0$ for $t<t_{0}, \delta_{I} \geq 0$, and $\varepsilon_{I}>0$.

Economic forces trying to bring the market into a new equilibrium position are described by the following ordinary differential equations with regard to the volumes of product supply $V_{S}(t)$, demand $V_{D}(t)$, and price $P(t)$ given the accumulated amounts of debt $S_{D}(t)$, debt servicing cost $S_{S}(t)$, and investment $S_{I}(t)$ on the market,

$$
\begin{aligned}
& \frac{d P(t)}{d t}=-\lambda_{P}\left(V_{S}(t)-V_{D}(t)-S_{D}(t)+S_{S}(t)-S_{I}(t)\right) \\
& \frac{d^{2} V_{S}(t)}{d t^{2}}=\lambda_{S} \frac{d P(t)}{d t} \\
& \frac{d^{2} V_{D}(t)}{d t^{2}}=-\lambda_{D} \frac{d^{2} P(t)}{d t^{2}}
\end{aligned}
$$


One may observe that a difference between the volumes of product demand and supply on the market is increasing, in the sense:

$$
V_{D}(t)-V_{S}(t)+\left(S_{I}(t)+S_{D}(t)-S_{S}(t)\right) \geq V_{D}(t)-V_{S}(t)
$$

if the sum of accumulated amount of investment $S_{I}(t)$ and accumulated amount of debt $S_{D}(t)$ exceeds the accumulated amount of debt servicing cost $S_{S}(t): S_{I}(t)+S_{D}(t) \geq S_{S}(t)$.

On the other hand, a difference between the volumes of product demand and supply on the market is decreasing, in the sense:

$$
V_{D}(t)-V_{S}(t)+\left(S_{I}(t)+S_{D}(t)-S_{S}(t)\right)<V_{D}(t)-V_{S}(t)
$$

if the sum of accumulated amount of investment $S_{I}(t)$ and accumulated amount of debt $S_{D}(t)$ goes below the accumulated amount of debt servicing cost $S_{S}(t): S_{I}(t)+S_{D}(t)<S_{S}(t)$.

Thus, if $\delta_{S} \delta_{D}>\varepsilon_{I}$ : in the time interval $t_{0} \leq t \leq t_{0}+\frac{2\left(\delta_{I}+\delta_{D}\right)}{\delta_{S} \delta_{D}-\varepsilon_{I}}$ an expansion of the product demand over supply takes place, and in the time interval $t_{0}+\frac{2\left(\delta_{I}+\delta_{D}\right)}{\delta_{S} \delta_{D}-\varepsilon_{I}}<t<+\infty$ a contraction of the product demand over supply happens. If $\delta_{S} \delta_{D} \leq \varepsilon_{I}$ then an expansion of the product demand over supply takes place in the entire time interval $t_{0} \leq t<+\infty$.

Let me introduce a new variable $D(t) \equiv\left(V_{S}(t)-V_{D}(t)-S_{D}(t)+S_{S}(t)-S_{I}(t)\right)$ representing the volume of product surplus (or shortage) on the market. Therefore, behavior of $D(t)$ is described by the following equation for $t>t_{0}$,

$$
\frac{d^{2} D(t)}{d t^{2}}+\lambda_{P} \lambda_{D} \frac{d D(t)}{d t}+\lambda_{P} \lambda_{S} D(t)+\varepsilon_{I}-\delta_{S} \delta_{D}=0
$$


with the initial conditions, $D\left(t_{0}\right)=0, \frac{d D\left(t_{0}\right)}{d t}=-\delta_{I}-\delta_{D}$.

If one uses another variable $D_{1}(t) \equiv D(t)+\frac{\varepsilon_{I}-\delta_{S} \delta_{D}}{\lambda_{P} \lambda_{S}}$, then Eq. (27) becomes,

$\frac{d^{2} D_{1}(t)}{d t^{2}}+\lambda_{P} \lambda_{D} \frac{d D_{1}(t)}{d t}+\lambda_{P} \lambda_{S} D_{1}(t)=0$

with the initial conditions, $D_{1}\left(t_{0}\right)=\frac{\varepsilon_{I}-\delta_{S} \delta_{D}}{\lambda_{P} \lambda_{S}}, \frac{d D_{1}\left(t_{0}\right)}{d t}=-\delta_{I}-\delta_{D}$.

Similar to Eq. (27), the product price $P(t)$ is described by the following equation for $t>t_{0}$,

$\frac{d^{2} P(t)}{d t^{2}}+\lambda_{P} \lambda_{D} \frac{d P(t)}{d t}+\lambda_{P} \lambda_{S}\left(P(t)-P^{0}-\frac{\delta_{I}+\delta_{D}}{\lambda_{S}}-\frac{\varepsilon_{I}-\delta_{S} \delta_{D}}{\lambda_{S}}\left(t-t_{0}\right)\right)=0$

with the initial conditions, $P\left(t_{0}\right)=P^{0}, \frac{d P\left(t_{0}\right)}{d t}=0$.

Let me introduce variable $P_{1}(t) \equiv P(t)-P^{0}-\frac{\delta_{I}+\delta_{D}}{\lambda_{S}}-\frac{\varepsilon_{I}-\delta_{S} \delta_{D}}{\lambda_{S}}\left(t-t_{0}\right)+\frac{\lambda_{D}}{\lambda_{S}^{2}}\left(\varepsilon_{I}-\delta_{S} \delta_{D}\right)$ to simplify an analysis of the product price behavior. The behavior of variable $P_{1}(t)$ is described by the following equation for $t>t_{0}$,

$\frac{d^{2} P_{1}(t)}{d t^{2}}+\lambda_{P} \lambda_{D} \frac{d P_{1}(t)}{d t}+\lambda_{P} \lambda_{S} P_{1}(t)=0$

with the initial conditions, $P_{1}\left(t_{0}\right)=-\frac{\delta_{I}+\delta_{D}}{\lambda_{S}}+\frac{\lambda_{D}}{\lambda_{S}^{2}}\left(\varepsilon_{I}-\delta_{S} \delta_{D}\right), \frac{d P_{1}\left(t_{0}\right)}{d t}=-\frac{\varepsilon_{I}-\delta_{S} \delta_{D}}{\lambda_{S}}$.

The behavior of solutions for $D_{1}(t)$ and $P_{1}(t)$ described by Eqs. (28) and (30) depends on the roots of the corresponding characteristic equations (Piskunov, 1965; Petrovski, 1966). Also Eqs. (28) and (30) have the same characteristic equations. 
When the roots of characteristic equation are complex-valued (i.e., $\frac{\lambda_{P}^{2} \lambda_{D}^{2}}{4}<\lambda_{P} \lambda_{S}$ ) both the variable $D_{1}(t)$ and variable $P_{1}(t)$ experience damped oscillations for time $t \geq t_{0}$. When the roots of characteristic equation are real and different (i.e., $\left.\frac{\lambda_{P}^{2} \lambda_{D}^{2}}{4}>\lambda_{P} \lambda_{S}\right)$ both the variable $D_{1}(t)$ and variable $P_{1}(t)$ don't oscillate for time $t \geq t_{0}$. When the roots of characteristic equation are real and equal (i.e., $\left.\frac{\lambda_{P}^{2} \lambda_{D}^{2}}{4}=\lambda_{P} \lambda_{S}\right)$ both the variable $D_{1}(t)$ and variable $P_{1}(t)$ don't oscillate for time $t \geq t_{0}$ as well.

It takes place $D_{1}(t) \rightarrow 0$ and $P_{1}(t) \rightarrow 0$ for $t \rightarrow+\infty$ if roots of characteristic equations are complexvalued $\left(\frac{\lambda_{P}^{2} \lambda_{D}^{2}}{4}<\lambda_{P} \lambda_{S}\right)$, real and different $\left(\frac{\lambda_{P}^{2} \lambda_{D}^{2}}{4}>\lambda_{P} \lambda_{S}\right)$, or real and equal $\left(\frac{\lambda_{P}^{2} \lambda_{D}^{2}}{4}=\lambda_{P} \lambda_{S}\right)$.

It takes place for the product surplus (shortage) $D(t)$, for the product price $P(t)$, for the product demand $V_{D}(t)$, for the product supply $V_{S}(t)$, for the amount of debt $S_{D}(t)$, for the amount of debt servicing cost $S_{S}(t)$, and for the amount of investment $S_{I}(t)$ if $t \rightarrow+\infty$,

$$
\begin{aligned}
& D(t) \rightarrow-\frac{\varepsilon_{I}-\delta_{S} \delta_{D}}{\lambda_{P} \lambda_{S}} \\
& P(t) \rightarrow \frac{\varepsilon_{I}-\delta_{S} \delta_{D}}{\lambda_{S}}\left(t-t_{0}\right)+P^{0}+\frac{\delta_{I}+\delta_{D}}{\lambda_{S}}-\frac{\lambda_{D}}{\lambda_{S}^{2}}\left(\varepsilon_{I}-\delta_{S} \delta_{D}\right) \\
& V_{D}(t) \rightarrow\left(r_{D}^{0}-\frac{\lambda_{D}}{\lambda_{S}}\left(\varepsilon_{I}-\delta_{S} \delta_{D}\right)\right)\left(t-t_{0}\right)+V_{D}^{0}-\frac{\lambda_{D}}{\lambda_{S}}\left(\delta_{I}+\delta_{D}\right)+\frac{\lambda_{D}^{2}}{\lambda_{S}^{2}}\left(\varepsilon_{I}-\delta_{S} \delta_{D}\right) \\
& V_{S}(t) \rightarrow\left(r_{D}^{0}+\delta_{I}+\delta_{D}-\frac{\lambda_{D}}{\lambda_{S}}\left(\varepsilon_{I}-\delta_{S} \delta_{D}\right)\right)\left(t-t_{0}\right)+\frac{\varepsilon_{I}-\delta_{S} \delta_{D}}{2}\left(t-t_{0}\right)^{2} \\
& +V_{D}^{0}-\frac{\lambda_{D}}{\lambda_{S}}\left(\delta_{I}+\delta_{D}\right)-\frac{\varepsilon_{I}-\delta_{S} \delta_{D}}{\lambda_{P} \lambda_{S}}+\frac{\lambda_{D}^{2}}{\lambda_{S}^{2}}\left(\varepsilon_{I}-\delta_{S} \delta_{D}\right)
\end{aligned}
$$




$$
\begin{aligned}
& S_{I}(t)=\delta_{I}\left(t-t_{0}\right)+\frac{\varepsilon_{I}}{2}\left(t-t_{0}\right)^{2} \\
& S_{D}(t)=\delta_{D}\left(t-t_{0}\right) \\
& S_{S}(t)=\frac{\delta_{S} \delta_{D}}{2}\left(t-t_{0}\right)^{2}
\end{aligned}
$$

To analyze an economic growth I use the variable $E_{D}(t) \equiv P(t) \times r_{D}(t)$ where $r_{D}(t) \equiv \frac{d V_{D}(t)}{d t}$, i.e., a rate of nominal demand for the product, which roughly represents the product earning on the market.

I compare the variable $E_{D}(t)$, a rate of nominal demand changed by the amount of debt $S_{D}(t)$, the amount of debt servicing cost $S_{S}(t)$, and the amount of investment $S_{I}(t)$, with the variable $\tilde{E}_{D}(t)$, original rate unchanged by the amounts of debt, debt servicing cost and investment, for $t \rightarrow+\infty$,

$$
\begin{aligned}
& E_{D}(t) \rightarrow\left(\frac{\varepsilon_{I}-\delta_{S} \delta_{D}}{\lambda_{S}}\left(t-t_{0}\right)+P^{0}+\frac{\delta_{I}+\delta_{D}}{\lambda_{S}}-\frac{\lambda_{D}}{\lambda_{S}^{2}}\left(\varepsilon_{I}-\delta_{S} \delta_{D}\right)\right)\left(r_{D}^{0}-\frac{\lambda_{D}}{\lambda_{S}}\left(\varepsilon_{I}-\delta_{S} \delta_{D}\right)\right) \text { and } \\
& \tilde{E}_{D}(t) \rightarrow P^{0} r_{D}^{0} .
\end{aligned}
$$

If the amount of debt $S_{D}(t)$ is increasing with a constant rate $\delta_{D}>0$ and the amount of investment $S_{I}(t)$ is increasing with a constant-acceleration $\varepsilon_{I}>0$ then resulting outcome depends on relationship between the acceleration of debt servicing cost $S_{S}(t): \delta_{S} \delta_{D}>0$, and the acceleration of investment $S_{I}(t): \varepsilon_{I}>0$

If $\delta_{S} \delta_{D}>\varepsilon_{I}$, the amount of debt servicing cost $S_{S}(t)$ is going to exceed the amount of investment $S_{I}(t)$ and ultimately causes an unrestricted decrease of the rate of nominal demand $E_{D}(t)$ with the passage of time. 
We can estimate a decrease $e_{D}(t)$ of the rate of nominal demand $E_{D}(t)$ where $e_{D}(t) \equiv \frac{d E_{D}(t)}{d t}$, i.e., the decrease of the rate of nominal demand for product, which roughly represents the decrease of the product earning on market.

It takes place, for $t \rightarrow+\infty, e_{D}(t) \rightarrow \frac{\varepsilon_{I}-\delta_{S} \delta_{D}}{\lambda_{S}}\left(r_{D}^{0}-\frac{\lambda_{D}}{\lambda_{S}}\left(\varepsilon_{I}-\delta_{S} \delta_{D}\right)\right)<0$ when $\delta_{S} \delta_{D}>\varepsilon_{I}$.

The limitary value of variable $e_{D}(t)$ doesn't have extremal points in the region $\delta_{S} \delta_{D}>\varepsilon_{I}$. In fact, the variable $e_{D}(t)$ has a maximal limitary value when $\varepsilon_{I}=\delta_{S} \delta_{D}+\frac{\lambda_{S}}{2 \lambda_{D}} r_{D}^{0}$. Respectively for $t \rightarrow+\infty$ $\max \left\{e_{D}(t)\right\} \rightarrow \frac{1}{4 \lambda_{D}}\left(r_{D}^{0}\right)^{2}>0$

Therefore, the limitary value of variable $e_{D}(t)$ is always negative in the region $\delta_{S} \delta_{D}>\varepsilon_{I}$. Maximum for the change of a rate of nominal demand for product, $\max \left\{e_{D}(t)\right\}$, is achieved outside of the region $\delta_{S} \delta_{D}>\varepsilon_{I}$, and is equal at the limit, for time $t \rightarrow+\infty, \max \left\{e_{D}(t)\right\} \rightarrow \frac{1}{4 \lambda_{D}}\left(r_{D}^{0}\right)^{2}>0$.

If $\delta_{S} \delta_{D}<\varepsilon_{I}<\delta_{S} \delta_{D}+\frac{\lambda_{S}}{\lambda_{D}} r_{D}^{0}$, the amount of investment $S_{I}(t)$ is going to exceed the amount of debt servicing cost $S_{S}(t)$ and $r_{D}(t)>0$. It ultimately causes an unrestricted increase of the rate of nominal demand $E_{D}(t)$ with the passage of time.

We can estimate an increase $e_{D}(t)$ of the rate of nominal demand $E_{D}(t)$, i.e., the increase of a rate of nominal demand for product, which roughly represents the increase of the product earning on market. 
It takes place $e_{D}(t) \rightarrow \frac{\varepsilon_{I}-\delta_{S} \delta_{D}}{\lambda_{S}}\left(r_{D}^{0}-\frac{\lambda_{D}}{\lambda_{S}}\left(\varepsilon_{I}-\delta_{S} \delta_{D}\right)\right)>0$ when $\delta_{S} \delta_{D}<\varepsilon_{I}<\delta_{S} \delta_{D}+\frac{\lambda_{S}}{\lambda_{D}} r_{D}^{0}$, for $t \rightarrow+\infty$.

The variable $e_{D}(t)$ has a maximal limitary value when $\varepsilon_{I}=\delta_{S} \delta_{D}+\frac{\lambda_{S}}{2 \lambda_{D}} r_{D}^{0}$. Then respectively for $t \rightarrow+\infty, \max \left\{e_{D}(t)\right\} \rightarrow \frac{1}{4 \lambda_{D}}\left(r_{D}^{0}\right)^{2}>0$.

Therefore, the maximal increase in a rate of nominal demand $\max \left\{e_{D}(t)\right\}$ for product on market, which roughly represents a maximal increase of the product earning or a maximal economic growth, is equal for time $t \rightarrow+\infty, \max \left\{e_{D}(t)\right\} \rightarrow \frac{1}{4 \lambda_{D}}\left(r_{D}^{0}\right)^{2}>0$

If $\delta_{S} \delta_{D}+\frac{\lambda_{S}}{\lambda_{D}} r_{D}^{0}<\varepsilon_{I}<+\infty$, the amount of investment $S_{I}(t)$ is going to exceed the amount of debt servicing cost $S_{S}(t)$ and $r_{D}(t)<0$. It ultimately causes an unrestricted decrease of the rate of nominal demand $E_{D}(t)$ with the passage of time.

We can estimate a decrease $e_{D}(t)$ of the rate of nominal demand $E_{D}(t)$, i.e., the decrease of a rate of nominal demand for product, which roughly represents a decrease of the product earning on market.

It takes place $e_{D}(t) \rightarrow \frac{\varepsilon_{I}-\delta_{S} \delta_{D}}{\lambda_{S}}\left(r_{D}^{0}-\frac{\lambda_{D}}{\lambda_{S}}\left(\varepsilon_{I}-\delta_{S} \delta_{D}\right)\right)<0$ when $\delta_{S} \delta_{D}+\frac{\lambda_{S}}{\lambda_{D}} r_{D}^{0}<\varepsilon_{I}<+\infty$, for $t \rightarrow+\infty$ 
In the region $\delta_{S} \delta_{D}+\frac{\lambda_{S}}{\lambda_{D}} r_{D}^{0}<\varepsilon_{I}<+\infty$ the limitary value of variable $e_{D}(t)$ doesn't have extremal points. In fact, the variable $e_{D}(t)$ has a maximal limitary value when $\varepsilon_{I}=\delta_{S} \delta_{D}+\frac{\lambda_{S}}{2 \lambda_{D}} r_{D}^{0}$. Hence respectively for $t \rightarrow+\infty, \max \left\{e_{D}(t)\right\} \rightarrow \frac{1}{4 \lambda_{D}}\left(r_{D}^{0}\right)^{2}>0$

Thus, the limitary value of variable $e_{D}(t)$ is always negative in the region $\delta_{S} \delta_{D}+\frac{\lambda_{S}}{\lambda_{D}} r_{D}^{0}<\varepsilon_{I}<+\infty$.

Maximum for the change of a rate of nominal demand for product, $\max \left\{e_{D}(t)\right\}$, is achieved outside of the region $\delta_{S} \delta_{D}+\frac{\lambda_{S}}{\lambda_{D}} r_{D}^{0}<\varepsilon_{I}<+\infty$, and is equal at the limit, $\max \left\{e_{D}(t)\right\} \rightarrow \frac{1}{4 \lambda_{D}}\left(r_{D}^{0}\right)^{2}>0$, for time $t \rightarrow+\infty$.

I will talk about economic implications of some results in the next section.

\section{Argumentative Economic Interpretation}

A single-product economy model of the credit expansion presented here can be briefly described as following. Initially, when the demand for product and supply of it are equal, the market is undisturbed.

The first scenario considered in the model is following. The demand for product on market is increased by assuming a constant-rate growing debt. Simultaneously, the supply of product on market was decreased by a constant-rate growing investment. The credit expansion in turn causes an increase of the debt servicing cost. Since the debt servicing cost is proportional to the accumulated amount of debt, i.e., an integral of the assumed debt over the time, the amount of debt servicing cost is growing with a constant acceleration. 
Thus, the sum of accumulated amount of investment and accumulated amount of debt grows with a constant rate and increases an excess of demand over supply. On the contrary, the accumulated amount of debt servicing cost grows with a constant acceleration and increases an excess of supply over demand. Eventually, the accumulated amount of debt servicing cost exceeds the sum of accumulated amount of investment and accumulated amount of debt. Hence, it takes place a limited short-term nominal economic growth initially and it takes place an unrestricted long-term nominal economic decline afterwards.

The second scenario considered in the model is following. The demand for product on market is increased by assuming a constant-rate growing debt. Simultaneously, the supply of product on market was decreased by a constant-acceleration growing investment. The credit expansion in turn causes an increase of the debt servicing cost. Since the debt servicing cost is proportional to the accumulated amount of debt, i.e., an integral of the assumed debt over the time, the amount of debt servicing cost is growing with a constant acceleration.

Thus, the sum of accumulated amount of investment and accumulated amount of debt grows with a constant acceleration and increases an excess of demand over supply. Likewise, the accumulated amount of debt servicing cost grows with a constant acceleration and increases an excess of supply over demand. Here, the logical outcome depends on quantitative values of two accelerations.

If the acceleration rate of debt servicing cost exceeds the acceleration rate of investment then the temporal axis is broken into two intervals. Initially, it takes place a limited short-term nominal economic growth. Afterwards, it takes place an unrestricted long-term nominal economic decline.

If the acceleration rate of investment exceeds the acceleration rate of debt servicing cost then the same process takes place over the whole temporal axis, and depends on the difference between two accelerations. If the acceleration rate of investment slightly exceeds the acceleration rate of debt servicing cost then it takes place an unrestricted long-term nominal economic growth. On the other hand, if the acceleration rate 
of investment considerably exceeds the acceleration rate of debt servicing cost then it takes place an unrestricted long-term nominal economic decline.

\section{Conclusions}

Presented here is a simplified mathematical model that investigates how economic concepts of investment, debt, demand and supply interact and make an impact on the nominal economic growth and decline. Two scenarios are considered. The first scenario assumes a constant-rate growing debt and a constant-rate growing investment. The scenario produces a limited short-term nominal economic growth initially and an unrestricted long-term nominal economic decline afterwards. The second scenario assumes a constant-rate growing debt and a constant-acceleration growing investment. When acceleration rate of debt servicing cost exceeds acceleration rate of investment the scenario delivers a limited short-term nominal economic growth initially and an unrestricted long-term nominal economic decline afterwards. When acceleration rate of investment slightly exceeds acceleration rate of debt servicing cost the scenario generates an unrestricted long-term nominal economic growth. When acceleration rate of investment considerably exceeds acceleration rate of debt servicing cost the scenario supplies an unrestricted long-term nominal economic decline.

The model is of a theoretical nature. It is rather an advanced one to be a simple economic tool but it is not fully developed to be able to solve practical economic tasks.

\section{References}

Krouglov, Alexei, 2006, Mathematical Dynamics of Economic Markets (New York: Nova Science Publishers).

Krouglov, Alexei, 2009, "Mathematical Dynamics of Economic Growth as Effect of Internal Savings," Finance India, Vol. 23, No. 1, pp. 99-136.

Krouglov, Alexei, "Secular Stagnation and Decline: A Simplified Model" (December 18, 2014). Available at SSRN: $\underline{\text { http://ssrn.com/abstract=2540408 }}$ or http://dx.doi.org/10.2139/ssrn.2540408. 
Krouglov, Alexei, "Credit Expansion and Contraction: A Simplified Model” (May 8, 2015). Available at SSRN: http://ssrn.com/abstract=2604176 or http://dx.doi.org/10.2139/ssrn.2604176.

Petrovski, Ivan G., 1966, Ordinary Differential Equations (Englewoods Cliffs, New Jersey: Prentice Hall).

Piskunov, Nikolai S., 1965, Differential and Integral Calculus (Groningen: P. Noordhoff). 